hep-ph/9606384

IASSNS-HEP-96/63

June 1996

BA-96-23

\title{
Gauged SO(3) Family Symmetry and Squark Mass Degeneracy ${ }^{\natural}$
}

\author{
K.S. Babu* and S.M. Barr ${ }^{\dagger}$ \\ *School of Natural Sciences \\ Institute for Advanced Study \\ Princeton, NJ 08540 \\ ${ }^{\dagger}$ Bartol Research Institute \\ University of Delaware \\ Newark, DE 19716
}

\begin{abstract}
It is shown that a gauged $\mathrm{SO}(3)$ family symmetry can suppress flavorchanging processes from squark-mass non-degeneracy to an acceptable level. The potentially dangerous $\mathrm{SO}(3)$ D-terms can be rendered harmless if the CPviolating phases appearing in the $\mathrm{SO}(3)$-breaking sector are small, which can naturally be the case if CP is a spontaneously broken symmetry. This approach has certain advantages over models based on global or non-abelian discrete symmetries, and dovetails with some recent proposals for explaining the pattern of quark and lepton masses. Moreover this approach relates the near-degeneracy of the squark masses to an approximate $\mathrm{CP}$ invariance which can also explain the smallness of the electron and neutron electric dipole moments.
\end{abstract}

\footnotetext{
${ }^{1}$ Research supported in part by DOE grants DE-FG02-90ER40542 and DE-FG02-91ER406267. Email: babu@sns.ias.edu, smbarr@bartol.udel.edu
} 


\section{Introduction}

Non-degeneracy of squark masses can lead to "flavor-changing neutral currents" (FCNC) that are unacceptably large [1]. In particular, the limits on the real and imaginary parts of the mass splitting between the $K_{L}$ and $K_{S}$ suggest a near degeneracy between the $\tilde{d}$ and $\tilde{s}$ squarks [2, 3]. Such a near degeneracy is hard to understand in the absence of some symmetry principle. The problem is made even more obscure by the fact that the quarks, far from exhibiting such a degeneracy, have a dramatic hierarchy of masses.

A symmetry rendering $\tilde{s}$ and $\tilde{d}$ nearly degenerate must be non-abelian. been proposed in the literature [6, 7, 8] that this role can be played by a family $\mathrm{SU}(2)$, or a discrete subgroup of it, under which the lightest two families form a doublet and the third generation forms a singlet. This is a structure consonant with both the presumed approximate degeneracy of $\tilde{s}$ and $\tilde{d}$ and the relatively large masses of $t, b$, and $\tau$.

An $\mathrm{SU}(2)$ under which the families form a $\mathbf{2}+\mathbf{1}$ cannot be gauged, as then the D-terms of $\mathrm{SU}(2)$ would directly contribute splittings to the squark masses comparable to the squark masses themselves. On the other hand, various arguments based on quantum gravity [10] seem to disfavor a global continuous symmetry below the Planck or string scale. This has led to the consideration of non-abelian discrete symmetry groups [9, 7]. However, since non-abelian discrete symmetries have up to now played little if any role in explaining particle phenomena, one may wonder if this is a completely satisfactory approach.

In this letter we shall discuss the possibility that the three families form a triplet of a gauged $\mathrm{SO}(3)$. Because the representations of $\mathrm{SO}(3)$ are real it turns out that the troublesome D-terms vanish for real vacuum expectation values. If $\mathrm{CP}$ is spontaneously broken then the $\mathrm{SO}(3)$-breaking VEVs can, indeed, naturally be approximately real. The squark-mass splittings then would be controlled by a naturally small $\mathrm{CP}$-violating phase. This is very appealing since there are independent grounds for suspecting that certain CP-violating phases must be small in supersymmetric theo-

\footnotetext{
${ }^{1}$ Degeneracy of squark masses could also arise if supersymmetry breaking is communicated to the squark sector via gauge interactions. For models based on this idea, see Ref. [1]. In this paper we shall be concerned with supersymmetry breaking via supergravity, in which case the squarks are not degenrate in general. For attempts to solve the squark-mediated FCNC problem using Abelian global symmetries, see Ref. [5].
} 
ries. In particular, the one-loop contributions to quark and electron electric dipole moments are small [11]. Moreover, the severest constraint on the non-degeneracy of $\tilde{d}$ and $\tilde{s}$ - namely from the $\epsilon_{K}$ parameter - is greatly alleviated if the relevant CP-violating phase is small [2, 3, 6, 7, 8, 9].

In the kinds of models we shall discuss, the large third-generation quark and lepton masses arise from a VEV which breaks $\mathrm{SO}(3)$ down to an approximate $\mathrm{SO}(2)$. As will be seen, in spite of the fact that $\mathrm{SO}(2)$ is abelian, the splitting of $\tilde{s}$ and $\tilde{d}$ is sufficiently controlled. Indeed, since the families form a $\mathbf{2}+\mathbf{1}$ (of course, reducible) under this residual $\mathrm{SO}(2)$, much of the discussion of squark mass splittings carries over with little change from the cases of $\mathrm{SU}(2)$ and its subgroups appearing in the literature.

Some recently proposed models [12] have shown that quark and lepton masses can be accounted for without using symmetries that distinguish among the generations all generations are treated on an equal footing [13]. In these models certain Yukawa couplings are vectors in "family space", that is they carry one family index. These models can be transposed very naturally into the context of a gauged family $\mathrm{SO}(3)$. In that transposition the Yukawa couplings that were vectors in family space are replaced by Higgs triplets of the $\mathrm{SO}(3)$. We will present an illustrative model that has a close affinity with that of Ref. [12], and which we believe shows certain advantages of $\mathrm{SO}(3)$ over other groups that have been discussed. In the model discussed in Section 4.2, most of the observed features of the quark and lepton masses are explained by various aspects of $\mathrm{SO}(10)$ gauge symmetry, while the squark mass degenracy is understood with a (commuting) $\mathrm{SO}(3)$ family gauge symmetry.

\section{The FCNC Problem and Squark-mass Degen- eracy}

Introducing low-energy supersymmetry produces several problems [1, 2, 6, 6, 11, 14] connected with flavor violation and $\mathrm{CP}$ violation that are absent in the Standard Model. For purposes of discussion it is convenient to distinguish four of them, even though in particular models they are closely related.

- (1) If the $\tilde{s}$ and $\tilde{d}$ masses are non-degenerate then mixing of $s$ and $d$ will lead, in general, to an excessively large $K_{L}-K_{S}$ mass splitting, $\Delta m_{K}$. For maximal

squark non-degeneracy $\operatorname{Re} M_{12}$ comes out about $10^{4}$ times too large [图, 迆. There 
are somewhat less dangerous contributions (by about an order of magnitude) also from mixing of $\tilde{b}$ with $\tilde{d}$.

- (2) The same effect, if CP-violating phases are present, leads to an excessively large $\operatorname{Im} M_{12}$, that is to a too large $\epsilon_{K}$. If the relevant phases are of order unity, then $\epsilon_{K}$ comes out about $10^{7}$ times too big [2, 3]. (The phases that are of most concern are those in the squark-mass matrix, although it should be kept in mind that $\mathrm{CP}$-phases in the quark-mass matrix can show up in the gluino box diagram when one rotates the phase of the $s$ quark to make the $K^{0} \rightarrow \pi \pi(I=0)$ amplitude real.)

- (3) Even with "universal" soft SUSY-breaking terms (i.e. degenerate squark masses and proportionality of $A$ terms) the electric dipole moment of the neutron will receive contributions at one (gluino) loop if the $A$ parameter or the $\mu$ parameter are complex. If these phases are of order unity, $d_{n}$ comes out typically about $10^{2}$ times too big [11, 3].

- (4) Finally, even if $A$ and $\mu$ are real, non-proportionality of the $A$-terms of the squarks to the quark Yukawa couplings gives a one-gluino-loop contribution to $d_{n}$ if there are phases in the quark mass matrix. If these phases are of order unity (as they need to be for the Kobayashi-Maskawa mechanism to work) typically $d_{n}$ is about $10^{2}$ too big [14].

It should be noted that these problems have somewhat different origins. Problems (1) and (2) arise from non-degeneracy of squark masses. Problems (2) and (3) arise from CP-violating phases in the soft terms that break supersymmetry. Problem (4) comes from non-proportionality of the $A$ terms.

It is with problems (1) and (2) that we are primarily concerned in this paper. They are quantitatively the most severe and would seem to suggest a high degree of degeneracy of the squarks (especially $\tilde{d}$ and $\tilde{s}$ ) no matter how CP is broken in the theory. (Indeed, problem (1) is a CP-conserving effect.) Our purpose in this paper is to propose an attractive explanation for this near-degeneracy. This explanation entails that certain CP-violating phases be small, which in turn suggests that $\mathrm{CP}$ is spontaneously broken. This fits in well with the requirements for solving problems (2) and (3), namely that the phases in the soft terms be small. However, neither squark degeneracy nor small phases in the soft terms will solve problem (4). The solution 
of problem (4) requires further conditions on the theory, in particular on the sector that generates quark and lepton masses. This issue will be discussed in Section 6 . For most of this paper, when we discuss the FCNC problem we will mean problems (1) and (2).

It should be noted that even if the squarks are non-degenerate at a high scale $M_{\mathrm{GUT}}$ (or $M_{\mathrm{Pl}}$ ), some amount of degeneracy could arise in the process of renormalization group running from $M_{\mathrm{GUT}}$ to the weak scale. This is a consequence of the gaugino loop corrections to the squark masses, which are flavor-blind. Similarly, the $A$ terms could become proportional to the Yukawa couplings to some extent as a result of the RGE flow, even though they are non-proportional at $M_{\mathrm{GUT}}$. The squark masses and the $A$ parameters at the weak scale (for the lighter two families) are given by

$$
\begin{aligned}
m_{Q_{i}}^{2}\left(m_{Z}\right) & \simeq m_{Q_{i}}^{2}\left(M_{\mathrm{GUT}}\right)+7.2 M_{1 / 2}^{2} \\
A_{i}\left(m_{Z}\right) & \simeq A_{i}\left(M_{\mathrm{GUT}}\right)-4.1 M_{1 / 2} .
\end{aligned}
$$

A $10 \%$ degeneracy in the squark mass-squared is not unreasonable to expect from the RGE flow (e.g: if $M_{Q_{i}}\left(M_{\mathrm{GUT}}\right) \sim M_{1 / 2}$ ), but this by itself is not sufficient to explain the constraints from the Kaon system. Similarly a $10 \%$ proportionality of the $A$ terms and the Yukawa couplings is quite plausible (e.g: if $\left.A_{i}\left(M_{\mathrm{GUT}}\right) \lesssim M_{1 / 2}\right)$. Since problem (4) noted above requires this proportionality to hold only to about $1 \%$, it appears to us that (4) is a much less severe problem.

The FCNC problem puts the most severe constraints on the degeneracy of the $\tilde{s}$ and $\tilde{d}$. Let us define $m_{Q}$ and $m_{D}$ to be the $L L$ and $R R$ mass matrices of the down squarks, respectively. And let $V^{Q}$ and $V^{D}$ be the unitary transformations that take the squark mass matrices from the basis where the quark mass matrices are real and diagonal and where the gluino couplings are flavor-conserving to the basis where $m_{Q}$ and $m_{D}$ are real and diagonal. Then the constraint from the $K_{L}-K_{S}$ mass splitting is (for $\left.m_{Q} \simeq m_{\tilde{g}}\right)$ [3]

$$
\left(\frac{\mathrm{TeV}}{m_{Q}}\right)^{2}\left|\frac{V_{11}^{Q} \delta m_{Q}^{2} V_{21}^{Q}}{m_{Q}^{2}} \frac{V_{11}^{D} \delta m_{D}^{2} V_{21}^{D}}{m_{D}^{2}}\right|<3.1 \times 10^{-3} .
$$

The constraint from $\epsilon_{K}$ is that

$$
\left(\frac{\mathrm{TeV}}{m_{Q}}\right)^{2}\left|\frac{V_{11}^{Q} \delta m_{Q}^{2} V_{21}^{Q}}{m_{Q}^{2}} \frac{V_{11}^{D} \delta m_{D}^{2} V_{21}^{D}}{m_{D}^{2}}\right| \sin \phi<1.9 \times 10^{-7},
$$


where $\phi \equiv \arg \left(V_{11}^{Q} V_{21}^{Q *} V_{11}^{D} V_{21}^{D *}\right)$.

From mixing with $\tilde{b}$ one has the condition (again from $\epsilon_{K}$ ) that

$$
\left(\frac{1 \mathrm{TeV}}{m_{\tilde{b}}}\right)^{2}\left|V_{13}^{Q} V_{23}^{Q} V_{13}^{D} V_{23}^{D}\right| \sin \phi^{\prime}<5 \times 10^{-8}
$$

If $\tilde{d}$ and $\tilde{s}$ had maximal non-degeneracy, that is if $\delta m_{Q, D}^{2} / m_{Q, D}^{2} \simeq 1$, and the phase $\phi$ were of order unity, then Eq. (3) would require that $m_{Q} \approx 200 \mathrm{TeV}$.

In the models under discussion in this paper (and also those in Ref. [7) one has that

$$
m_{Q}^{2}, m_{D}^{2}=\left(\begin{array}{ccc}
1 & \lambda^{3} & \lambda^{3} \\
\lambda^{3} & 1+\lambda^{2} & \lambda^{2} \\
\lambda^{3} & \lambda^{2} & O(1)
\end{array}\right) m^{2},
$$

where $\lambda^{n}$ means $\mathrm{O}\left(\lambda^{n}\right)$, and where $\lambda=\sin \theta_{C} \simeq .22$ is the Wolfenstein parameter. That means that in Eq. (2), $\delta m_{Q, D}^{2} / m_{Q, D}^{2} \sim \lambda^{2}$ and $V_{21}^{Q, D} \sim \lambda$, and thus the condition there is easily satisfied for squark masses of order $100 \mathrm{GeV}$. The condition from the $\epsilon_{K}$ parameter, however, gives (Eq. (3)) that $m_{Q}>10^{3} \lambda^{3} \sqrt{\sin \phi} \mathrm{TeV} \simeq 24 \mathrm{TeV} \sqrt{\sin \phi}$, which implies that $\phi$ is of order $10^{-3}$ or $10^{-4}$. And in Eq. (4), $V_{13}^{Q, D} \sim \lambda^{3}, V_{23}^{Q, D} \sim \lambda^{2}$, so that $m_{Q}>4.5 \times 10^{3} \lambda^{5} \sqrt{\sin \phi^{\prime}} \mathrm{TeV} \sim 2.3 \sqrt{\sin \phi^{\prime}} \mathrm{TeV}$. Thus $\phi^{\prime}$ must also be small. (As noted parenthetically where "problem (2)" was defined above, phases in the quark-mass matrix also contribute to $\epsilon_{K}$ indirectly through the gluino box diagram. In particular, the most dangerous contribution comes from a phase in the (12) or (21) elements of the quark-mass matrices. These also give the most dangerous contribution to problem (4). Thus whatever solves problem (4) tends to eliminate or lessen this problem as well. See the discussion in Sec. 6.)

To see how such a form as Eq. (5) may be achieved using non-abelian family symmetry, it is helpful to review, at least in outline, a model proposed in Ref. [7].

Consider a model with low-energy supersymmetry and the family group $G_{H}$, which is either $S U(2) \times U(1) \times Z_{2}$ or some discrete subgroup thereof. The families are arranged in $\mathbf{2}+\mathbf{1}$ representations of the non-abelian group: $\left(Q+Q_{3}\right),\left(d^{c}+d_{3}^{c}\right)$, and $\left(u^{c}+u_{3}^{c}\right)$, where the representations without the subscript ' 3 ' are understood to be doublets under the family group, and to contain the first two generations. Breaking the non-abelian family group is a doublet, $\Phi$, which has VEV given by $\langle\Phi\rangle=\Lambda(0, \epsilon)^{T}$. $\Lambda$ is a scale characterizing new physics that is represented by higher-dimensional operators involving $\Phi$ appearing in an effective lagrangian. The doublets, $Q, d^{c}$, and 
$u^{c}$, all have charge 1 under the $\mathrm{U}(1)$, while the doublet $\Phi$ has charge -1 . The Higgs field $\Phi$ is odd under the $Z_{2}$ as is a singlet Higgs field $\chi$.

The third generation fermions are heavy because their masses arise from dimensionfour terms:

$$
\mathcal{L}_{Y}^{0}=h_{d} d_{3}^{c} H_{d} Q_{3}+h_{u} u_{3}^{c} H_{u} Q_{3} .
$$

The next layer of the "onion structure" of the mass matrices is provided by the higher-dimension terms

$$
\mathcal{L}_{Y}=h_{d}\left(d^{c T}, d_{3}^{c}\right)\left(\begin{array}{cc}
h_{\Phi \Phi}^{d} \Phi \Phi^{T} / \Lambda^{2} & h_{\Phi \chi}^{d} \Phi \chi / \Lambda^{2} \\
h_{\chi \Phi}^{d} \Phi^{T} \chi / \Lambda^{2} & 1
\end{array}\right)\left(\begin{array}{c}
Q \\
Q_{3}
\end{array}\right) H_{d}+\{d \rightarrow u\}+h . c . .
$$

The singlet field $\chi$ has $\operatorname{VEV}\langle\chi\rangle=\Lambda \epsilon_{\chi}$, where $\epsilon_{\chi}$ is of the same order as $\epsilon$. This gives the following structure to the down-quark mass matrix;

$$
M_{d}=\left(\begin{array}{ccc}
0 & 0 & 0 \\
0 & h_{\Phi \Phi}^{d} \epsilon^{2} & h_{\Phi \chi}^{d} \epsilon \epsilon_{\chi} \\
0 & h_{\chi \Phi}^{d} \epsilon \epsilon_{\chi} & 1
\end{array}\right) h_{d}\left\langle H_{d}\right\rangle,
$$

with a similar form for the up-quark and charged lepton mass matrices. From the fact that $V_{c b}$ and $m_{s} / m_{b}$ are roughly the same and of order $\lambda^{2}$ one sees that $\epsilon \simeq \epsilon_{\chi} \sim \lambda$. However, this fact is not explained in this framework, since the fields $\chi$ and $\Phi$ have different symmetry properties, and therefore there is no obvious reason why $\epsilon$ and $\epsilon_{\chi}$ should be comparable. The $\mathrm{SO}(3)$ models, as we shall see, can do better in this regard. There are several ways to generate the first generation masses and mixings, but these are not of immediate concern to us.

The leading contribution to the squark-masses come from effective operators [7, 15] of the form

$$
\mathcal{L}_{\text {soft }}=\int d^{4} \theta Q^{\dagger} Q \bar{\eta} \eta
$$

and others with $Q$ replaced by $Q_{3}, d^{c}, d_{3}^{c}$ and so on. $\eta$ is a dimensionless "spurion" superfield which represents the effect of supersymmetry breaking. $\langle\eta\rangle=m_{S U S Y} \theta^{2}$. The family symmetry, $\mathrm{SU}(2)$, or a discrete non-abelian subgroup of it, ensures that there is a degeneracy between the sparticle masses of the first two generations.

There are a number of contributions to the non-degeneracy of $\tilde{s}$ and $\tilde{d}$. One is from higher-dimension operators of the form [7, 15]

$$
\mathcal{L}_{\text {soft }}^{\prime}=\int d^{4} \theta Q^{\dagger} \Phi \Phi^{\dagger} Q \bar{\eta} \eta / \Lambda^{2}
$$


From the form of the VEV of $\Phi$, and the structure of the mass matrix $M_{d}$, it is readily apparent that the effect of such a term is to generate a contribution to the mass-squared for $\tilde{s}$ that is of order $\lambda^{2} m_{S U S Y}^{2}$. In other words, the fractional splitting between the $\tilde{d}$ and $\tilde{s}$ masses is of order $10^{-2}$. This is true both of the splitting in the left-squark sector (given by the expression in Eq. (10)) and of the splitting in the right-squark sector (given by the analogous expression with $Q$ replaced by $d^{c}$ ). There are other contributions to the squark mass splittings, but they are no larger in order of magnitude than this.

\section{$3 \quad \mathrm{SO}(3)$ Breaking and the Effect of the D-Terms}

Consider a gauged $\mathrm{SO}(3)$ symmetry broken spontaneously by a set of M vectors: $\vec{A}_{i}$, $i=1, \ldots, M$. Assume that the left and right-handed (s)quarks and (s)leptons are all in vector representations of $\mathrm{SO}(3)$, which we will generically denote by $\vec{\phi}_{n}$. The $\mathrm{SO}(3)$ $\mathrm{D}$ term in the potential then has the form

$$
V_{D}\left(\vec{A}_{i}\right)=\sum_{a=1}^{3}\left(D^{a}\right)^{2}=\left|\sum_{n} \vec{\phi}_{n}^{*} \times \vec{\phi}_{n}+\sum_{i} \vec{A}_{i}^{*} \times \vec{A}_{i}\right|^{2} .
$$

Note that the generators of $\mathrm{SO}(3)$ in the vector representation can be written using the familiar cross product. It is clear that if the VEVs that spontaneously break $\mathrm{SO}(3)$ contribute non-vanishingly to the $\mathrm{SO}(3)$ D-term, the cross terms in Eq. (11) will lead to intergenerational splittings of the squark masses. It is this that has made gauged continuous family symmetries disfavored as a solution to the squark-mass nondegeneracy problem. However, it is obvious that if $\mathrm{CP}$ were conserved, the VEVs of the vectors $\overrightarrow{A_{i}}$ would be real and would contribute nothing to the dangerous D-terms, since $\vec{A}_{i}^{*} \times \vec{A}_{i}=2 i \vec{A}_{i R} \times \vec{A}_{i I}$.

Since the $\mathrm{SO}(3)$ D-terms break supersymmetry, they must be proportional to $m_{S U S Y}$. Thus one expects that the splittings among the mass-squared of the squarks due to these D-terms will be of order $\theta_{C P} m_{S U S Y}^{2}$, where $\theta_{C P}$ represents the magnitude of the CP-violating phases appearing in the potential of the vectors $\vec{A}_{i}$. Since the overall magnitude of the squark masses is itself of order $m_{S U S Y}$, the D-term contributions to the fractional splittings $\delta m_{Q}^{2} / m_{Q}^{2}$ and $\delta m_{D}^{2} / m_{D}^{2}$ appearing in Eqs. (2) and (3) are of order $\theta_{C P}$. In a theory in which CP is spontaneously broken, these phases can be naturally small. 
To be more explicit, let us assume that there are three vectors, $\vec{A}_{i}, i=1,2,3$, which break $\mathrm{SO}(3)$ spontaneously at a scale $\Lambda$ which is near the Planck scale. Since we will assume that the superpotential for these fields has CP-violating phases which are very small, we can expand the VEVs of the vectors $\vec{A}_{i}$ in powers of this $\mathrm{CP}$ violation. In particular, we can write

$$
\left\langle\vec{A}_{i}\right\rangle=\vec{A}_{i}^{(0)}+i \vec{b}_{i}+O\left(\theta_{C P}^{2}\right)
$$

where $\vec{A}_{i}^{(0)}$ is the VEV in the limit that the CP-violating phases are set to zero. The imaginary parts of the VEVs, $\vec{b}_{i}$, are thus of order $\theta_{C P}$.

Clearly, if we neglect the soft, supersymmetry-breaking terms that are of order the weak scale, the VEVs of the $\vec{A}_{i}$ must make what remains of the potential, namely the F-terms and D-terms, vanish — otherwise supersymmetry would break at the scale $\Lambda \sim M_{P l}$. The F terms can only depend on the $\vec{A}_{i}$ through the six $\operatorname{SO}(3)$ invariant complex quantities $\overrightarrow{A_{i}} \cdot \overrightarrow{A_{j}}$. Thus their vanishing gives 12 real conditions to be satisfied. (Another way to argue this, which applies to any number, M, of vectors, is that the $\vec{A}_{i}$ contain $6 M$ real quantities, but the superpotential, being holomorphic, is invariant under the complex extension of $\mathrm{SO}(3)$, and so 6 of the parameters are left undetermined by the $\mathrm{F}$ terms. Thus $6(M-1)$, in our case 12 , conditions are imposed by the $\mathrm{F}$ terms.) These can be written to leading order in $\mathrm{CP}$ violation

$$
\begin{aligned}
& \vec{A}_{i}^{(0)} \cdot \vec{b}_{i} \quad=\left|\vec{A}_{i}^{(0)}\right|^{2} \theta_{i} \\
& \vec{A}_{i}^{(0)} \cdot \vec{b}_{j}+\vec{A}_{j}^{(0)} \cdot \vec{b}_{i}=\left|\vec{A}_{i}^{(0)} \cdot \vec{A}_{j}^{(0)}\right| \theta_{i j}, \quad i \neq j,
\end{aligned}
$$

where $\theta_{i}$ and $\theta_{i j}$ are combinations of CP-violating phases appearing in the superpotential, and are of order $\theta_{C P}$.

The D-terms depend only on the real three-vector $\vec{D}$, and so their vanishing gives 3 more real conditions, which can be written to leading order in CP-violation as

$$
\sum_{i} \vec{A}_{i}^{(0)} \times \vec{b}_{i}=0
$$

But the three vectors $\vec{A}_{i}$ have 18 real degrees of freedom, of which three are gauge degrees of freedom. Thus there are 15 conditions to be satisfied by 15 parameters, and there is in general a non-trivial solution to Eqs. (13) - (14). This solution can be expressed schematically as $b \sim \theta_{C P} A^{(0)}$. The D-term in the potential can be written in the same schematic way as $V_{D} \sim\left|g A^{(0)}\left(b-\theta_{C P} A^{(0)}\right)+g \phi_{n}^{*} \tau \phi_{n}\right|^{2} \cdot g$ is the $\mathrm{SO}(3)$ 
gauge coupling constant, and $\tau$ is an $\mathrm{SO}(3)$ generator. When the soft supersymmetrybreaking terms are added, one has effectively a potential for $\vec{b}_{i}$ that can be written as $V(b) \sim g^{2}\left(A^{(0)}\right)^{2}\left(b-\theta_{C P} A^{(0)}\right)^{2}+m_{0}^{2}|b|^{2}$. This gives $g^{2}\left(A^{(0)}\right)^{2}\left(b-\theta_{C P} A^{(0)}\right) \sim m_{0}^{2} b \sim$ $\theta_{C P} m_{0}^{2} A^{(0)}$. Therefore the contribution to the squark and slepton mass splittings are of order $g^{2} A^{(0)}\left(b-\theta_{C P} A^{(0)}\right)\left(\phi_{n}^{*} \tau \phi_{n}\right) \sim \theta_{C P} m_{0}^{2}\left(\phi_{n}^{*} \tau \phi_{n}\right)$. This verifies our earlier claim that the fractional squark-mass splittings due to the $\mathrm{SO}(3)$ D-terms are simply of order the CP-violating phases that appear in the superpotential of the vectors that break $\mathrm{SO}(3)$. Observe that the $\mathrm{SO}(3)$ gauge coupling drops out.

A more careful treatment can be done that gives the order in $\lambda$ of the contributions to the different squark-mass-matrix elements coming from these $\mathrm{SO}(3)$ D-terms. Suppose that $\left|\overrightarrow{A_{1}}\right|^{2} \sim 1,\left|\overrightarrow{A_{2}}\right|^{2} \sim \lambda^{2}$, and $\left|\overrightarrow{A_{3}}\right|^{2} \sim \lambda^{3}$, and that $\overrightarrow{A_{1}}$ lies approximately in the ' 3 ' direction in family space, $\overrightarrow{A_{2}}$ lies approximately in the '2-3 plane', and $\overrightarrow{A_{3}}$ has components in all three directions, with the direction cosines $\hat{A}_{i} \cdot \hat{A}_{j}$ all being of order unity. (These conditions apply to the types of models described in the next section.) Then it can be shown that the $\mathrm{SO}(3)$ D-term contributions to the squark masses are of order $\theta_{C P} m_{S U S Y}^{2}\left(O\left(\lambda^{2}\right), O\left(\lambda^{3}\right), O(\lambda)\right) \cdot \vec{Q}^{\dagger} \times \vec{Q}$. In particular, writing $\vec{Q}=\left(\tilde{d}_{L}, \tilde{s}_{L}, \tilde{b}_{L}\right)$

$$
\vec{Q}^{\dagger} m_{Q}^{2} \vec{Q}=\left(\tilde{d}_{L}, \tilde{s}_{L}, \tilde{b}_{L}\right)^{*}\left(\begin{array}{ccc}
1 & \lambda^{3}+i \theta_{C P} \lambda & \lambda^{3}+i \theta_{C P} \lambda^{3} \\
\lambda^{3}-i \theta_{C P} \lambda & 1+\lambda^{2} & \lambda^{2}+i \theta_{C P} \lambda^{2} \\
\lambda^{3}-i \theta_{C P} \lambda^{3} & \lambda^{2}-i \theta_{C P} \lambda^{2} & O(1)
\end{array}\right)\left(\begin{array}{c}
\tilde{d}_{L} \\
\tilde{s}_{L} \\
\tilde{b}_{L}
\end{array}\right)
$$

where, as before, $\lambda^{n}$ means $O\left(\lambda^{n}\right)$. The real parts come from other sources, to be discussed later. The imaginary (CP-violating) parts come from the $\mathrm{SO}(3)$ D-terms. There are similar matrices for $\vec{D}^{c}=\left(\tilde{d}_{L}^{c}, \tilde{s}_{L}^{c}, \tilde{b}_{L}^{c}\right)$.

Since we know that to satisfy the condition on the real part of $M_{12}$ in the Kaon system (Eq. (2)) the $\tilde{d}^{*} \tilde{s}$ and $\tilde{s}^{*} \tilde{d}$ elements of $m_{Q, D}^{2}$ must be $O\left(\lambda^{3}\right)$, one sees from Eq. (15) that $\theta_{C P}$ must also be no larger than $O\left(\lambda^{2}\right)$. Moreover, from Eq. (15) it is easy to see that the phase $\phi$ in Eq. (3) is $\arg \left\{\left[O\left(\lambda^{3}\right)+i O\left(\theta_{C P} \lambda\right)\right]^{2}\right\} \sim \theta_{C P} / \lambda^{2}$. Thus, to satisfy the condition on $\epsilon_{K}$ one needs $\theta_{C P} \sim 10^{-4}$.

It is noteworthy that $\mathrm{CP}$-violating effects from squark mixing involving the third generation is more highly suppressed. The constraint arising from the ( $\mathrm{CP}$-conserving) $B_{d}-\bar{B}_{d}$ system is

$$
\left(\frac{\mathrm{TeV}}{m_{Q}}\right)^{2}\left|\frac{V_{11}^{Q} \delta m_{Q}^{2} V_{31}^{Q}}{m_{Q}^{2}} \frac{V_{11}^{D} \delta m_{D}^{2} V_{31}^{D}}{m_{D}^{2}}\right|<1.3 \times 10^{-3}
$$


From the form of Eq. (15), noting that $\delta m_{Q}^{2} / m_{Q}^{2} \sim \mathcal{O}(1)$ for the $\tilde{b}-\tilde{d}$ system, we see that the quantity on the left-hand side of Eq. (16) is approximately $\lambda^{6} \sim 1 \times 10^{-4}$. This clearly satisfies the constraint. As for the $\mathrm{CP}$-violating effects, note that they arise with additional factors of $\theta_{C P}$ and are thus extremely small.

\section{Types of Models}

We may distinguish two approaches to constructing realistic models using an $\mathrm{SO}(3)$ family gauge symmetry. What we will call Approach I is similar in effect to models with abelian continuous or discrete family symmetries that distinguish among the generations. This approach can lead to "texture" models of fermion masses. The main difference here is that in our case we are using gauged $\mathrm{SO}(3)$ symmetry to arrive at such forms. Approach II is similar in effect to models based on "factorization" of Yukawa terms 116, 13, 12.

\subsection{Approach I: Texture models}

Let there be a set of vectors which break $\mathrm{SO}(3)$ spontaneously. We will call them $\vec{A}, \vec{B}$, $\vec{C}$, etc. (instead of $\vec{A}_{i}$ as heretofore). One can imagine that the superpotential of these vectors is such as to make the VEVs of any pair of them either orthogonal or parallel. Each vector can be taken to define a direction in "family space" corresponding to a particular generation (before diagonalizing the fermion mass matrices). For example, $\vec{A}$ might be the longest vector and be involved in giving mass to the heavy third generation through effective terms like $\vec{A} \cdot \vec{D}^{c} \vec{A} \cdot \vec{Q} H$, etc. Thus $\vec{A}$ would define the ' 3 ' direction in family space, and this term would be $A^{2} d_{3}^{c} Q_{3} H$. Since the VEVs of $\vec{B}$ and $\vec{C}$ are orthogonal to that of $\vec{A}$ and to each other, they can be taken to define the ' 2 ' and ' 1 ' directions in family space. Thus a (21) element would come from $\vec{B} \cdot \vec{D}^{c} \vec{C} \cdot \vec{Q} H=B C\left(d_{2}^{c} Q_{1}\right) H$.

A set of discrete symmetries that distinguish among the vectors $\vec{A}, \vec{B}, \vec{C}$, etc. would effectively be equivalent to a set of discrete symmetries that distinguish family generation number. Consider, for example, a set of $Z_{2}$ symmetries, $K_{A} \times K_{B} \times K_{C}$, where $K_{A}: \vec{A} \longrightarrow-\vec{A}, \vec{B} \longrightarrow+\vec{B}, \vec{C} \longrightarrow+\vec{C}$, and similarly for $K_{B}$ and $K_{C}$. If

\footnotetext{
${ }^{2}$ Fairly simple superpotentials lead to this result. For example, the superpotential $W=\sum_{i=1}^{3} \vec{A}_{i}$. $\vec{A}_{i}+\sum_{i, j=1}^{3}\left(\vec{A}_{i} \cdot \vec{A}_{j}\right)^{2}$ has a SUSY preserving minimum where $\vec{A}_{3} \sim(0,0,1), \overrightarrow{A_{2}} \sim(0,1,0), \overrightarrow{A_{1}} \sim$ $(1,0,0)$.
} 
one had a scalar field that had parity $(-,-,+)$ under $K_{A} \times K_{B} \times K_{C}$ then it could couple to $\vec{B} \cdot \vec{D}^{c} \vec{A} \cdot \vec{Q}$ and $\vec{A} \cdot \vec{D}^{c} \vec{B} \cdot \vec{Q}$ and therefore in the (23) and (32) elements of mass matrices but not in others. If this scalar is a GUT non-singlet (such as a $\mathbf{4 5}$ of $\mathrm{SO}(10)$ ), it will not upset the desired VEV pattern of the three $\mathrm{SO}(3)$ vectors. In such a way "texture" models could be constructed of the quark and lepton mass matrices. So, although one does not actually have an abelian family symmetry $Z_{2} \times Z_{2} \times Z_{2}$ one achieves the same result. More complicated discrete family symmetries can be simulated in the same way.

It should also be noted that the kind of discrete symmetry suggested above could explain why the vectors $\vec{A}, \vec{B}, \vec{C}$, etc., have VEVs that are orthogonal or parallel. For example, a term in the superpotential of the form $M \vec{A} \cdot \vec{B}$ would be forbidden, and $(\vec{A} \cdot \vec{B})^{2} / M$ allowed. If terms of yet higher dimension can be neglected then $\theta_{A B}=n \pi / 2$. On the other hand, if both these terms were present $\theta_{A B}$ could take non-trivial values.

As an example of an $\mathrm{SO}(10)$ model in this approach, consider the couplings

$W=(\overrightarrow{\mathbf{1 6}} \cdot \vec{A}) \overline{\mathbf{1 6}}_{A}+(\overrightarrow{\mathbf{1 6}} \cdot \vec{B}) \overline{\mathbf{1 6}}_{B}+(\overrightarrow{\mathbf{1 6}} \cdot \vec{C}) \overline{\mathbf{1 6}}_{C}+\sum_{\alpha=A, B, C} \overline{\mathbf{1 6}}_{\alpha} \mathbf{1 6}{ }_{\alpha}+\sum_{\alpha=A, B, C} \mathbf{1 6} \mathbf{6}_{\alpha} \mathbf{1 6} \mathbf{1 0}$ where $\overline{\mathbf{1 6}}_{A}$ carries $K_{A}$ charge etc. For generation mixing, we utilize the couplings $\overline{\mathbf{1 6}}_{A} \mathbf{1 6}_{B} \mathbf{4 5} \mathbf{5}_{A B}$ where $\mathbf{4 5} \mathbf{5}_{A B}$ has both $K_{A}$ and $K_{B}$ charges, etc. When the $\overline{\mathbf{1 6}}_{A, B, C}+$ $\mathbf{1 6}_{A, B, C}$ are integrated out, light fermion mass matrices with specific textures will emerge.

\subsection{Approach II: Factorization models}

Another way to explain the hierarchy of quark and lepton masses that has been proposed in the literature [16, 13, 12] and that does not involve abelian family symmetry, is the idea of the "factorization" of fermion mass matrices. If the Yukawa couplings are matrices in family space, like $\sum_{i j} F_{i j}\left(Q_{i}^{c} Q_{j} H\right)$, then one expects mass matrices of rank 3 with no particular hierarchy among their eigenvalues. On the other hand, if one has a factorized form like $\sum_{i j} F_{i} F_{j}^{\prime}\left(Q_{i}^{c} Q_{j} H\right)$, then no matter in what direction $F_{i}$ and $F_{j}^{\prime}$ point the mass matrix is rank 1 . If this were the dominant term it would explain why one family is much heavier than the others. A second (by assumption smaller) term $G_{i} G_{j}^{\prime}\left(Q_{i}^{c} Q_{j} H\right)$ would, together with the previous term, automatically give a mass matrix of rank 2 (unless $G \| F$ or $G^{\prime} \| F^{\prime}$ ). And similarly a third term 
would give mass to the remaining generation. The crucial point is that no special relative directions or absolute directions for the vectors $F_{i}, G_{i}$, etc., need be assumed. In a sense, the basic idea of factorization is opposite to that of abelian continuous or discrete family symmetry: the whole idea is not to specify a priori special directions in family space.

The way such factorized forms arise in the models proposed in the literature [16, 13, 12] is through integrating out heavy fields. For example, if there are the terms $M \Psi^{c} \Psi+F_{i} \Psi^{c} Q_{i}\langle\Omega\rangle+F_{i}^{\prime} d_{i}^{c} \Psi\langle H\rangle$, where $M$ and $\langle\Omega\rangle$ are comparable and large, then integrating out $\Psi^{c}+\Psi$ gives the effective term $F_{i} F_{j}^{\prime}(\langle\Omega\rangle / M) Q_{i} d_{j}^{c} H$.

It is easy to see that one may implement this factorization idea readily in the context of SO(3) family gauge symmetry. One simply has to promote the Yukawa couplings that are vectors in family space to be the VEVs of Higgs fields that are triplets of $\mathrm{SO}(3)$. One actually achieves more flexibility in doing this, because in the models [16, 13, 12] where the Yukawa vectors are just numbers, they must have (barring fine-tuning) arbitrary relative directions, whereas here more than one "Yukawa" vector could come from the same $\mathrm{SO}(3)$ Higgs triplet and so be aligned.

Here we will present an $\mathrm{SO}(10)$ model with $\mathrm{SO}(3)$ gauge family symmetry that is similar to that proposed in Ref. [12], which explains in an economical way many features of the quark and lepton spectrum, and which solves the problem of FCNC coming from squark-mass non-degeneracy. For helpful background the reader is referred to Ref. 12.

In this example model, as in Ref. [12], the nontrivial pattern of quark and lepton masses is a consequence of the mixing of the three families with new vectorlike familyantifamily pairs. In particular, we posit the existence of an $\mathrm{SO}(3)$ triplet of families, $\overrightarrow{\mathbf{1 6}}$, and a set of fields which are singlets under $\mathrm{SO}(3)$ but transform under $\mathrm{SO}(10)$ as the vectorlike pairs $\mathbf{1 6}+\overline{\mathbf{1 6}}$, and $\mathbf{1 6}^{\prime}+\overline{\mathbf{1 6}}^{\prime}$, and the real representations $\mathbf{1 0}+\mathbf{1 0}^{\prime}$. Involved in the Yukawa terms of the quarks and leptons are the Higgs fields $\mathbf{4} \mathbf{5}_{H}$, $\mathbf{4 5} X, \mathbf{4 5}_{B-L}, \mathbf{1 6} \mathbf{6}_{H}$, and $\mathbf{1 0} \mathbf{0}_{H}$. The two adjoint Higgs point respectively in the $X$ and $B-L$ directions, where $X$ is the $\mathrm{SU}(5)$-singlet generator. (The field $\mathbf{4 5} \mathbf{5}_{H}$ which is assumed to have a VEV in a general direction need not be distinct from $4 \mathbf{5}_{X}$, but its VEV cannot be strictly along the $B-L$ direction, see below.) The $\mathbf{1 6}_{H}$ gets a GUT-scale VEV in the SU(5)-singlet direction and a weak-scale VEV in its ordinary weak-doublet component. The $\mathbf{1 0}_{H}$ also breaks the weak interactions. 
The Yukawa terms of the quarks and leptons are as follows:

$$
\begin{aligned}
& \mathcal{L}_{\text {Yukawa }}=a_{0} M \mathbf{1 6} \overline{\mathbf{1 6}}+(\vec{a} \cdot \overrightarrow{\mathbf{1 6}}) \overline{\mathbf{1 6}}\left\langle\mathbf{4 5} 5_{H}\right\rangle+(\vec{a} \cdot \overrightarrow{\mathbf{1 6}}) \mathbf{1 6}\left\langle\mathbf{1 0}_{H}\right\rangle \\
& b_{0} \mathbf{1 6} \overline{\mathbf{1 6}}^{\prime}\left\langle 45_{X}\right\rangle+(\vec{b} \cdot \mathbf{1 6}) \overrightarrow{\mathbf{1 6}}^{\prime}\left\langle\mathbf{4 5} 5_{B-L}\right\rangle+\left(\overrightarrow{b^{\prime}} \cdot \overrightarrow{\mathbf{1 6}}\right) \mathbf{1 6} \mathbf{6}^{\prime}\left\langle\mathbf{1 0} 0_{H}\right\rangle
\end{aligned}
$$

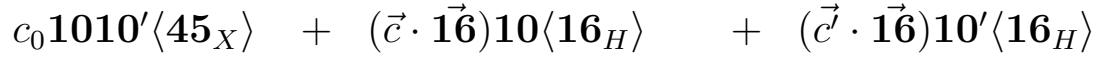

The vectors $\vec{a}, \vec{b}, \overrightarrow{b^{\prime}}, \vec{c}$, and $\overrightarrow{c^{\prime}}$ at this level are just coupling constants which explicitly break $\mathrm{SO}(3)$, but they are to be understood as arising from the VEVs of $\mathrm{SO}(3)$-vector Higgs fields in an $\mathrm{SO}(3)$-invariant theory. The same vector $\vec{a}$ appears in two terms, and can be taken without loss of generality to point in the ' 3 ' direction. (These vectors are real, for reasons explained above, to order $10^{-4}$.) The two vectors $\vec{b}$ and $\overrightarrow{b^{\prime}}$ are assumed to be coplanar with $\vec{a}$ and can without loss of generality be taken to lie in the '2-3 plane'. The vector $\vec{c}$ (and/or $\overrightarrow{c^{\prime}}$ ) has a component in the ' 1 ' direction. This pattern will be shown to arise naturally from the underlying theory with $\mathrm{SO}(3)$ family symmetry.

The first set of three terms in Eq. (17) generates mass for the third generation. Integrating out the $\mathbf{1 6}+\overline{\mathbf{1 6}}$ gives an effective term of the form $(Q \vec{a} \cdot \overrightarrow{\mathbf{1 6}})(\vec{a} \cdot \overrightarrow{\mathbf{1 6}})\left\langle\mathbf{1 0}_{H}\right\rangle / a_{0}$, where $Q$ is the generator of $\mathrm{SO}(10)$ that tells which direction $\left\langle\mathbf{4} \mathbf{5}_{H}\right\rangle$ points in. It is easy to show [12 that, no matter what $Q$ is, $m_{b}=m_{\tau}$ at the GUT scale. The second set of three terms generates the next layer of the onion, namely masses for the second generation. Integrating out the $\mathbf{1 6}^{\prime}+\overline{\mathbf{1 6}}^{\prime}$ one obtains an effective operator approximately (for small mixing) of the form $\left(\frac{B-L}{X} \vec{b} \cdot \mathbf{1} \mathbf{6}\right)\left(\overrightarrow{b^{\prime}} \cdot \overrightarrow{\mathbf{1 6}}\right)$. For fermions of type $f$ $(f=u, d, l)$ this term gives $\left(M_{f}\right)_{i j} \propto\left(\frac{B-L}{X}\right)_{f^{c}} b_{i} b_{j}^{\prime}+\left(\frac{B-L}{X}\right)_{f} b_{j} b_{i}^{\prime}$. For the down-quark and charged-lepton mass matrices, this gives non-zero contributions to the (22), (23), (32), and (33) elements that are all of the same order (assuming that the vectors $\vec{b}$ and $\vec{b}^{\prime}$ point in arbitrary directions in the (23) plane). The second generation masses, then, come predominantly from the (22) elements, and $V_{c b} \sim m_{s} / m_{b} \sim \lambda^{2}$. Moreover, because of the factor of $B-L$, the entries in the lepton mass matrix are three times as large as those in the down-quark mass matrix. This gives the Georgi-Jarlskog [17] factor of 3 between the $\mu$ and $s$ masses.

On the other hand, because $X$ is the same for $u$ and $u^{c}$, while $B-L$ is opposite in sign, it is obvious that the contribution of this term to the up-quark mass matrix is anti-symmetric. Therefore the (22) element of $M_{u}$ vanishes, and the $c$ mass comes from mixing with the third generation. Thus $m_{c} / m_{t} \sim \lambda^{4} \ll m_{s} / m_{b}$, as observed.

Finally, the last set of three terms in Eq. (17) generates the first generation masses and mixings. It is easily shown that the effective operator gotten from integrating out 
the $10+\mathbf{1 0}^{\prime}$ is (if mixings are small) antisymmetric [12]. This can be seen from the fact that, because the $\mathbf{4 5}$ is antisymmetric, the effective operator changes sign when one interchanges the $\mathbf{1 0}$ and $\mathbf{1 0} \mathbf{0}^{\prime}$. This also interchanges $\vec{c}$ and $\overrightarrow{c^{\prime}}$ and thus the rows and columns of the mass matrices. This antisymmetric contribution is only to the down-quark and lepton mass matrices. Because the $\mathbf{1 0}$ of $\mathrm{SO}(10)$ does not contain up quarks, there is no contribution from this effective operator to the up-quark mass matrix.

One has therefore accounted for several more facts. The relative smallness of $m_{u} / m_{t}$ is a consequence of the up-quark matrix still being rank-two at this level. The facts that the (11) elements vanish and that the (12) and (21) elements are equal in magnitude lead both to the familiar relation $\tan \theta_{C} \cong \sqrt{m_{d} / m_{s}}$, and to the Georgi-Jarlskog factor of $\frac{1}{3}$ between the $e$ and $d$ masses (since $\operatorname{Det}\left(M_{d}\right) \simeq \operatorname{Det}\left(M_{l}\right)$ ).

Let us now turn to the underlying $\mathrm{SO}(3)$-invariant theory. There must be at least three $\mathrm{SO}(3)$-vector Higgs, which again shall be called $\vec{A}, \vec{B}, \vec{C}$, etc.. The Yukawa coupling constant $\vec{a}$ comes from the VEV of $\vec{A}$. So the second term in Eq. (17) comes from a higher-dimension term $(a / \Lambda)(\vec{A} \cdot \overrightarrow{\mathbf{1 6}}) \overline{\mathbf{1 6}} 45_{X} . \quad a$ is just some dimensionless coupling, and $\Lambda \sim M_{P l}$. Similarly, for the third term in Eq. (17). Because the effective Yukawa coupling of the top quark is about 1 , it is easily seen that $\langle\vec{A}\rangle \approx \Lambda$. The Yukawa constants, $\vec{b}, \overrightarrow{b^{\prime}}, \vec{c}$, and $\overrightarrow{c^{\prime}}$ come from the VEVs of $\vec{B}$ and $\vec{C}$. Because these lead to smaller quark and lepton masses, it is natural to assume that these VEVs are somewhat smaller than $\Lambda$. For example, from the earlier discussion one expects that $|\langle\vec{B}\rangle|^{2} / \Lambda^{2} \sim m_{s} / m_{b} \sim \lambda^{2}$.

Clearly, there must be some symmetry that distinguishes the three fields $\vec{A}, \vec{B}$, and $\vec{C}$, or else they would couple indiscriminately and destroy the specific pattern in Eq. (17). A simple possibility is $K_{A} \times K_{B} \times K_{C}$, where each $K$ is a $Z_{2}$ which reflects one of the vectors. By means of this symmetry one can ensure that at the dimension-four level only the vector $\vec{B}$ appears in the fifth and sixth term of Eq. (17). However, at the dimension-six level one can have also $\left(\vec{B} \cdot \vec{A} / \Lambda^{2}\right) \vec{A}$, which is of the same order as $\vec{B}$, and has the same transformation properties under $K_{A} \times K_{B} \times K_{C}$. Thus the effective Yukawa couplings in the fifth and six terms of Eq. (17) will be (different) linear combinations of $\vec{B}$ and $\vec{A}$. Vectors $\vec{a}, \vec{b}$, and $\vec{b}^{\prime}$ will be approximately coplanar, and the angles between them will be order unity, as desired. (There will also be contributions of order $\left(\vec{B} \cdot \vec{C} / \Lambda^{2}\right) \vec{C}$, but these will be negligibly small.)

In the eighth and ninth terms of Eq. (17) one must have $\vec{C}$ and another vector 
$\vec{D}$ (which could be $\vec{B}$ ) at the dimension-four level. Then $\vec{c}$ arises predominantly from $\vec{C}$ and $\left(\vec{C} \cdot \vec{A} / \Lambda^{2}\right) \vec{A}$, and lies approximately in the $A-C$ plane. Similarly, $\overrightarrow{c^{\prime}}$ arises predominantly from $\vec{D}$ and $\left(\vec{D} \cdot \vec{A} / \Lambda^{2}\right) \vec{A}$ and lies approximately in the $A-D$ plane. With this arrangement the full rank-three mass matrices of the quarks and leptons are generated. From the magnitude of the first generation masses it is reasonable to assume that $|\langle\vec{C}\rangle \times\langle\vec{D}\rangle| \sim \lambda^{3}$.

It is apparent that the scenario outlined above requires that the VEVs of the vectors $\vec{A}, \vec{B}, \vec{C}$, etc. must have some non-trivial relative orientation; they cannot be nearly parallel or nearly orthogonal. Superpotentials leading to this situation are easy to construct. Consider, for example, the angle between $\vec{A}$ and $\vec{B}$. As noted above, if there are both linear and quadratic terms in $(\vec{A} \cdot \vec{B})$ then a nontrivial angle will in general result. However, while the term $(\vec{A} \cdot \vec{B})^{2} / \Lambda$ is allowed by $K_{A} \times K_{B}$, $M \vec{A} \cdot \vec{B}$ is not. This problem is overcome if there are singlet fields, $A_{0}$ and $B_{0}$, that are odd under $K_{A}$ and $K_{B}$ respectively, and whose VEVs are of the same order as $\langle\vec{A}\rangle$ and $\langle\vec{B}\rangle$. Then a term of the form $A_{0} B_{0}(\vec{A} \cdot \vec{B}) / \Lambda$ is allowed. Another possibility is to utilize the coupling $|\vec{A} \times \vec{B} \cdot \vec{C}|^{2}$ which is invariant under the discrete symmetries. This term will induce non-trivial angles between the vectors. Yet another possibility is to use a singlet $\phi$ which is odd under all three parity symmetries, this can have an invariant coupling $\vec{A} \times \vec{B} \cdot \vec{C} \phi$, which will again induce non-trivial angles between the vectors.

\section{The Lifting of the Squark-mass Degeneracy}

How big will the violations of squark-mass degeneracy be? We will discuss this in the context of the $\mathrm{SO}(10)$ example presented in the last section. The dominant contribution to the $\tilde{d}-\tilde{s}$ non-degeneracy, in complete analogy with the "SU(2)" models discussed in Sec. 2, comes from effective terms of the form

$$
\mathcal{L}_{\text {soft }}=\int d^{4} \theta \overrightarrow{\mathbf{1 6}}^{\dagger} \cdot \vec{B} \vec{B}^{\dagger} \cdot \overrightarrow{\mathbf{1 6}} \bar{\eta} \eta / \Lambda^{2}
$$

Since we know from the discussion of the last section that $|\langle\vec{B}\rangle|^{2} / \Lambda^{2} \sim m_{s} / m_{b} \sim \lambda^{2}$, it follows that this term will contribute $O\left(\lambda^{2}\right)$ to the (22), (23), (32), and (33) elements of the squark mass matrices, which is consistent with Eq. (4) and sufficiently small. This is the same result that obtains in the models based on $\mathrm{SU}(2)$ and its discrete subgroups. 
In the kinds of models described in the last section the large VEV of $\vec{A}$ breaks $\mathrm{SO}(3)$ down to an approximate $\mathrm{SO}(2)$. Since this is abelian one might wonder whether this residual symmetry will be sufficient to protect the near-degeneracy of the $\tilde{s}$ and $\tilde{d}$ squarks. The dangerous operator that is allowed by $\operatorname{SO}(3)$ (and which has no analogue in the models based on $\mathrm{SU}(2)$ and its subgroups) is

$$
\int d^{4} \theta\left(\overrightarrow{\mathbf{1 6}}^{\dagger} \cdot \vec{A} \times \overrightarrow{\mathbf{1 6}}\right) \bar{\eta} \eta / \Lambda
$$

While this is invariant under $\mathrm{SO}(3)$, it is odd under $K_{A}$. However, at least in the models of Approach II, an allowed and equally dangerous term is gotten by multiplying the above operator by $A_{0} / \Lambda$.

Fortunately, this dangerous class of operators can be forbidden by going to the gauge group $\mathrm{O}(3)$, instead of $\mathrm{SO}(3)$. In other words, by adding the $Z_{2}$ under which all vectors of $\mathrm{SO}(3)$ are odd. Then the dangerous operator of Eq. (19) can only be made even under this $Z_{2}$ by putting in an odd number of additional factors of $\mathrm{SO}(3)$-vector fields. But this, in turn, is only possible using the "triple product", $\vec{A} \cdot \vec{B} \times \vec{C}$. Hence, the lowest operator of the type shown in Eq. (19) is

$$
\int d^{4} \theta\left(\overrightarrow{\mathbf{1 6}}^{\dagger} \cdot \vec{A} \times \mathbf{1 \mathbf { 6 }}\right)(\vec{A} \cdot \vec{B} \times \vec{C}) B_{0} C_{0} \bar{\eta} \eta / \Lambda^{4}
$$

which is $O\left(\frac{B^{2}}{\Lambda^{2}} \frac{C^{2}}{\Lambda^{2}} m_{S U S Y}^{2}\right) \sim O\left(\lambda^{5}\right)$ and therefore negligible.

To avoid problems in the Kaon system it is also necessary to ensure that the mixing of $\tilde{b}$ with $\tilde{s}$ and $\tilde{d}$ be sufficiently small, and in particular of order $\lambda^{2}$ and $\lambda^{3}$ respectively. (See Eq. (5).) The $\tilde{b}-\tilde{s}$ mixing comes both from Eq. (16) (which we have seen is not dangerous) and from effective operators of the form

$$
\int d^{4} \theta\left(\overrightarrow{\mathbf{1 6}}^{\dagger} \cdot \vec{A}\right)(\vec{B} \cdot \mathbf{1 \mathbf { 6 }})\left(\vec{A} \cdot \vec{B} \text { or } A_{0} B_{0}\right) \bar{\eta} \eta / \Lambda^{4}
$$

Note that one had to go to order $\Lambda^{-4}$ to get an operator that is invariant under $K_{A} \times K_{B}$. This gives a result for the mixing that is of order $\lambda^{2}$, as required. (The result is even smaller in Approach I models where $\vec{A} \cdot \vec{B}$ vanishes (at least approximately) and where there is no need for the fields $A_{0}$ and $B_{0}$.)

The $\tilde{b}-\tilde{d}$ mixing comes from operators of the same form as Eq. (21) with $\vec{B}$ and $B_{0}$ replaced by $\vec{C}$ and $C_{0}$. The resulting mixing is typically of order $\lambda^{3}$ as required. 


\section{The Breaking of CP and Electric Dipole Mo- ments}

So far we have an explanation using spontaneously broken $\mathrm{SO}(3)$ and $\mathrm{CP}$ for the apparent near-degeneracy of the squarks of the first two generations. Since that explanation involves certain CP-violating phases being naturally small, one can get "for free", as it were, a solution to problems (2) and (3) discussed in section 2. However, this does not solve problem (4), since the family $\mathrm{SO}(3)$ does not make the A-terms proportional to the Yukawa terms. Moreover, even if the phases in the $\mathrm{SO}(3)$-breaking VEVs, the squark masses, the A parameter(s) and $\mu$ are naturally very small, the Kobayashi-Maskawa mechanism requires that some phase in the quark mass matrices be of order unity. (There is no conflict here with the assumption that $\mathrm{SO}(3)$ breaking VEVs are approximately real, since order one complex phases can arise from the $\mathrm{SO}(10)$ breaking sector.) This would lead in general to $d_{n}$ being about two orders of magnitude too large. As noted already, RGE running can introduce some proportionality, of order $10 \%$ or so between $A$ and the Yukawa couplings, so the tuning needed is only about $1 / 10$. This may not be a serious difficulty. Nevertheless, let us elaborate on some other alternatives to avoid problem (4).

There are several ways around this problem. The most radical is simply to abandon the Kobayashi-Maskawa mechanism. That is, one lets the phases in the quark mass matrices be small also. This does not create a problem in explaining $\epsilon_{K}$, since the gluino-box diagrams that were the source of problem (2) can do that - indeed we have had to assume that certain phases were about $10^{-4}$ to avoid $\epsilon_{K}$ being too large. However, these box diagrams will not give $\epsilon^{\prime} / \epsilon$ near the present experimental limit. If $\epsilon^{\prime}$ is indeed near the present limit, one would have to invoke some new mechanism. One possibility is that spontaneous $\mathrm{CP}$ violation occurring in the Weak scale Higgs sector could give milliweak-type effects. Since it is not necessary that these give a significant contribution to $\epsilon_{K}$ (which is already assumed to be taken care of by gluino loops) it would seem possible that Higgs effects could generate $\epsilon^{\prime}$ and $d_{n}$ of the right magnitudes.

It is also possible to maintain the Kobayashi-Maskawa explanation of the Kaonsystem CP violation. According to Ref. [14 one needs, in order to solve problem (4), that the quark mass matrices (and A-terms) have a special form. In particular in is

helpful if certain off-diagonal elements vanish [14]. But we have already seen that 
in Approach I models can be constructed with non-trivial "textures" for the fermion mass matrices.

It is somewhat trickier to solve problem (4) in models which are based on Approach II while maintaining the KM mechanism. However, it is possible to do so, as we shall show by a slight modification of the $\mathrm{SO}(10)$ model presented above.

Suppose that the effective Yukawa couplings $\vec{c}$ and $\overrightarrow{c^{\prime}}$ in Eq. (17) arise (at dimension-4 level) from the same $\mathrm{SO}(3)$ vector Higgs, $\vec{C}$. Then both $\vec{c}$ and $\overrightarrow{c^{\prime}}$ lie in the $\vec{A}-\vec{C}$ plane (approximately). To the extent that this approximation is good, the last three terms in Eq. (17) will only generate (13), (31), (23), and (32) elements. These elements of the down-quark mass matrix can have phases that are large, with reasonable values of squark masses, without leading to an excessive $d_{n}$ [14]. Thus by introducing a large phase into some VEV that appears only in one of the last three terms in Eq. (17), one can get a large KM phase, $\delta_{K}$, without creating a difficulty. However, a large phase in the (12) and (21) elements of the quark mass matrices would be fatal [14]. But with the new assumption we have made one can show that the (complex) contribution to these elements from the last three terms of Eq. (17) is only of order $\lambda^{5}$. One still needs to generate a real contribution to the (12) and (21) that is of order $\lambda^{3}$ to give first generation fermion masses and the Cabibbo angle. This can be done by introducing a new set of terms analogous to the last three in Eq. (17) but which do not have a large phase.

\section{Conclusions}

We have suggested in this paper that a gauged $\mathrm{SO}(3)$ family symmetry can cure the FCNC problem arising from the squark mass non-degeneracy. What enabled us to gauge the $\mathrm{SO}(3)$ symmetry is the near-vanishing of the $\mathrm{SO}(3)$ D-terms, which potentially could have contributed at an unacceptable level to the squark mass nondegeneracy. Since triplet representation of $\mathrm{SO}(3)$ is real, any non-zero D-term contributions to the squark mass splittings can only arise proportional to $\mathrm{CP}$-violating phases in the triplet VEVs. If $\mathrm{CP}$ violation has a spontaneous origin, which may be desirable from other considerations (EDM of the neutron and the electron), these phases can be naturally small. We showed how it is possible to transcribe certain recently suggested fermion mass schemes based on $\mathrm{SO}(10)$ symmetry into this $\mathrm{SO}(3)$ framework. The $\mathrm{SO}(3)$ family symmetry can also mimick Abelian symmetries en- 
abling one to construct texture models of fermion masses.

\section{Acknowledgments}

We wish to thank R. Rattazzi for stimulating discussions.

\section{References}

[1] S. Dimopoulos and H. Georgi, Nucl. Phys. B193, 150, 1981; L.J. Hall, V.A. Kostelecki, and S. Raby, Nucl. Phys. B267, 415 (1986); H. Georgi, Phys. Lett. B169, 231 (1986); S. Dimopoulos and D. Sutter, Nucl. Phys. B452, 496 (1995).

[2] J. Donoghue, H. Nilles, and D. Wyler, Phys. Lett. B128, 55 (1983); F. Gabbiani and A. Masiero, Nucl. Phys. B322, 235 (1989); J. Hagelin, S. Kelley, and T. Tanaka, Nucl. Phys. B415, 293 (1994).

[3] F. Gabbiani, E. Gabrielli, A. Masiero and L. Silvestrini, hep-ph/9604387.

[4] M. Dine and A. Nelson, Phys. Rev. D48, 1277 (1993); M. Dine, A. Nelson and Y. Shirman, Phys. Rev. D51, 1362 (1995); M. Dine, A. Nelson, Y. Nir and Y. Shirman, Phys. Rev. D53, 2658 (1996).

[5] Y. Nir and N. Seiberg, Phys. Lett. 309B, 337 (1993).

[6] P. Pouliot and N. Seiberg, Phys. Lett. 318B, 169 (1993); M. Dine, R. Leigh, and A. Kagan, Phys. Rev. D48, 4269 (1993); D.B. Kaplan and M. Schmaltz, Phys. Rev. D49, 3741 (1994).

[7] A. Pomarol and D. Tommasini, CERN-TH/95-207, hep-ph/9567462.

[8] R. Barbieri, G. Dvali and L. Hall, hep-ph/9512388.

[9] L. Hall and H. Murayama, Phys. Rev. Lett. 75, 3985 (1995); C. Carone, L. Hall and H. Murayama, hep-ph/9602364; P. Frampton and O. Kong, Phys. Rev. D53, 2293 (1996) and hep-ph/9603372; K.C. Chou and Y.L. Wu, Phys. Rev. D53, 3492 (1996)) and hep-ph/9603282.

[10] See, e.g., T. Banks, Physicalia 12, 19 (1990); J. Preskill, Proc. of the International Symposium on Gravity (The Woodlands, Texas, 1992). 
[11] J. Ellis, S. Ferrara, and D.V. Nanopoulos, Phys. Lett. B114, 231 (1982); W. Buchmuller and D. Wyler, Phys. Lett. B121, 321 (1982); J. Polchinski and M. Wise, Phys. Lett. B125, 393 (1983); F. del Aguila, M.B. Gavela, J.A. Grifols, and A. Mendez, Phys. Lett. B126, 71 (1983). For a review see S.M. Barr, Int. J. Mod. Phys. A8, 209 (1993).

[12] K.S. Babu and S.M. Barr, Phys. Rev. Lett. 75, 2088 (1995); K.S. Babu and S.M. Barr, Bartol Preprint BA-95-55, hep-ph/9512389.

[13] B.S. Balakrishna, A. Kagan and R.N. Mohapatra, Phys. Lett. 205B, 345 (1988); K.S. Babu and R.N. Mohapatra, Phys. Rev. Lett. 64, 2747 (1990); R.N. Mohapatra, in Proceedings of the Workshop on Fermion Masses, Fermilab, 1993; Z. Berezhiani, Phys. Lett. 129B, 99 (1983); J.C. Pati, Phys. Lett. B228, 228 (1989).

[14] R. Rattazzi, private communications (to be publsihed) and Y. Nir and R. Rattazzi, hep-ph/9603233.

[15] L. Girardello and M. Grisaru, Nucl. Phys. B194, 65 (1982); A. Pomarol and S. Dimopoulos, CERN-TH/95-114, hep-ph/9505302; R. Rattazzi, hep-ph/9507315.

[16] S.M. Barr, Phys. rev. D24, 1895 (1981); S.M. Barr, Phys. Rev. Lett. 64, 353 (1990), Phys. Rev. D42, 3150 (1990).

[17] H. Georgi and C. Jarlskog, Phys. Lett. 86B, 297 (1979). 